\title{
HEALTH-RELATED MOBILE APPLICATIONS FOR SMART DEVICES ON INSURANCE PROVIDERS: AIDING CUSTOMER RETENTION
}

\author{
Shravan Santosh Baratam, Harrisburg University of Science and Technology, SBaratam@my.harrisburgu.edu \\ Chaza F. Abdul, Harrisburg University of Science and Technology, cabdul@harrisburgu.edu \\ Loreen M. Powell, Bloomsburg University of Pennsylvania, lpowell@bloomu.edu
}

\begin{abstract}
This empirical research explores the number of healthcare insurance providers/payers using mobile applications for smart devices and analyzes the significance between the use of the mobile application for smart devices and their customer retention. A questionnaire was used to gather data. Forty-one employees from health insurance and private insurance providers completed the questionnaire. The results found that only $51.21 \%$ of healthcare insurance providers/payers utilize/require mobile applications and $75.61 \%$ plan to utilize/require mobile applications. A significant relationship was found between the use of mobile applications and customer retention; however, no significant correlation was found between gender and the use of mobile applications and customer retention. This work serves as an important step towards recognizing the business value aspects of health-related mobile applications for smart devices on insurance providers.
\end{abstract}

Keywords: Healthcare technology, health-related mobile applications, smart devices, health insurance providers, customer retention

\section{INTRODUCTION}

Today, the increase in diagnosed chronic diseases such as diabetes, cancer, heart failure and arthritis has contributed to the need for technological change which has assisted in the treatment of healthcare to diagnosed patients and the rise of healthcare costs (Amadeo, 2020). However, technology advances have made access to information affordable and easy to find for patients. Specifically, artificial intelligence (AI), Internet of things (IOT) and health-related mobile applications for smart devices are some of the most recent technology advances that offer enormous potential for the healthcare industry (Daecher et al., 2018; King 2017; Marakhimov \& Joo, 2017; Shah \& Chircu, 2018; Verweij \& Roa, 2017).

Additionally, there's been an explosion of health-related mobile applications in the last decade (Pinchot, 2018; Sydow, 2018) on smart devices. Suleymanova (2013) stated that in 2013 there were more than " 40,000 medical apps and 250 million downloads". Today, this number has substantially grown. Many of these health-related mobile applications on smart devices can assist the physician and the patient in treatment in hopes to speed up the process to get the patient as healthy as possible (Free et al. 2013; Egbedion, Wimmer, Rebman, \& Powell, 2019). The healthier the patient, the lower the cost is for healthcare insurance providers/payers. Hence, health-related mobile applications on smart devices can help insurance companies/payers to stay competitive by reducing costs, increase the member of acquisition and maintain members' retention. Thus, many researchers suggest that healthcare dealmakers/insurance providers should consider mobile tools as a medium to improve members' retention (Collins, 2014; Sulevymanova, 2103.)

There is an abundance of mobile technologies, and healthcare research exploring this phenomenon. However, the majority of the existing research tends to focus on the application development (Egbedion, et al. 2018), consumer adoption (Marakhimov \& Joo, 2017), benefits (MacLeod, 2018), usability, or security issues (Koohang et al, 2019; Sampat, \& Prabhakar, 2017; Tovino, 2019). However, there is little to no research found on United States (US) healthcare insurance providers'/payers' use or requirement of health-related mobile applications to communicate healthcare needs to its members. Specifically, there is a gap in the literature on the business value aspects of healthcare mobile applications such as customer retention. The purpose of this empirical study is to examine the number of health insurance providers utilizing/requiring health-related mobile applications for smart devices and the relationship between their use and customer retention. This work has practical implications for the health informatics field, 
healthcare insurance providers/payers and health-related mobile application developers. The remaining structure of this paper is as follows: brief review of the literature, research questions, methodology, results, and conclusion.

\section{BRIEF REVIEW OF LITERATURE}

In 2017, the US healthcare costs were over 3.5 trillion dollars (Amadeo, 2020). Looking at the size of the market, there are many demands and opportunities for innovation. One major area for growth and innovation is health-related technology advancements (Hilfiker, Santana, Freedman, \& Harris, 2019).

Currently, approximately $90 \%$ of Americans use the internet. Moreover, within the last three years, approximately $81 \%$ of Americans have searched available online sources for health-related content using a computer or a mobile device (Pew Research Center A., n.d.; Polansky, Leslie, \& Heimann, 2018). In 2019, a study estimated that approximately $62 \%$ of American smartphone users will use their devices to get information about a health condition (Pew Research Center B., n.d). Additionally, recent studies also show that the number of mobile users accessing health-related content is projected to increase as more households abandon a personal computer and Internet service and move towards an entirely mobile/smart phone use to access information online needs (Hilfiker, Santana, Freedman, \& Harris, 2019; Ray, Sewell, Gilbert and Roberts; 2017).

Currently, the mobile application market, in general, has exploded within the last few years (Pinchot, 2018). Healthrelated mobile applications are tools that offer its users various healthcare related functions. The fact that mobile devices, especially mobile phones are "personal, intelligent, connected, and always with people" (Fiordelli, Divani and Schulz, 2013), provide a large advantage within their use in healthcare. Smartphones in this era are highly equipped with features that help access and generate variety of data that is useful for both, the insurance providers and members (Clemens, 2017; Hilfiker, Santana, Freedman, \& Harris, 2019; Ray, Sewell, Gilbert and Roberts; 2017). In smartphone platforms such as Android and iOS, applications have an option to access various hardware features of the phone. Such features include the camera, GPS location, microphone, voice recognition, and texts in real time (Liu, Zhu, Holroyd, \& Seng, 2011). Health-related mobile applications can be found for just about every smart device.

Moreover, extensive recent research has been conducted on the positive benefits that healthcare related mobile applications for smart devices offer for patients. Specifically, mobile technologies have the penitential to be a lowcost opportunity for keeping participants informed and engaged about current research studies (Ray, Gilbert, \& Sewell; 2016), allowing healthcare organizations to maintain consistent contact with their customers (Ray et al., 2017), as well as collecting and tracking data on the customer. This can improve patient's overall health status (Clemens, 2017) and increase motivation in patients' populations (Morrison, Conway \& Yardley, 2013).

While healthcare apps have great potential and benefits, not everyone currently uses them. Mackert, Mabry-Flynn, Champlin, Donovan, and Pounders (2016) studied 4,974 American adults regarding health literacy and its association between patients' use of fitness and nutrition apps, activity trackers, and patient portals. Specifically, they examined the perceived ease of use, usefulness, and privacy of the technology tools/apps, as well as the trust in government, media, and technology companies and providers, and healthcare industry. They found significant differences $(\mathrm{p}>.05)$ among all factors examined. However most importantly they found that people with low health literacy were less likely to use these apps/tools and suggested that further research be conducted on health literacy and healthcare apps' adoption and usage.

Another recent study by Hilfiker et al (2019) reported that relying on customers to search the internet or utilize apps for health-related information is not effective as many people have limited literacy skills, or less technologically savvy. The authors argued that compounding literacy issues with complex health related content can make it very frustrating for end-users. As a result, many people presented great difficulty with website searches and navigation. This issue is a long-standing issue. In 2015 the U.S. Office of Disease Prevention and Health Promotion (ODPHP) released a guide (Health Literacy Online: A Guide for Simplifying the User Experience) to help with these difficulties. However, Hilfiker et al. (2019) explained that this problem still exists; however, they highlighted some strategies to consider when seeking to post healthcare information online that is useful for members and knowledge seekers. 
There are many another challenges beside literacy and usability skills. Hoffmann, Jansen, and Glasziou (2018) argued that providing accurate healthcare information for the elderly is difficult as many have various multimorbidity. They explained that information often found online doesn't explain that the elderly may be excluded for as a result of their multiple chronic illness/multimorbidity. Additionally, they explained that multimorbidity provides a barrier/exception to the rule for using typical guidelines or decisions aids found online. Hence, this is confusing to the patient as the information often conflicts with what appropriate care approaches, they should learn about.

\section{Business Value via Customer Retention}

Rama and Saritha (2019) stated that customer retention is one of the main challenges before the insurance providers. As a result, many customer relationship management (CRM) programs have been implemented. However, CRMs, in general, tend to "manage a company's interactions with current and future customers", and this "involves using technology to organize, automate, and synchronize sales, marketing, customer service, and technical support" (Rama \& Saritha, 2019).

Ehrenhard et al. (2016) explained that business value can be created from mobile applications. Specifically, mobile applications which could possibly reduce cost and increase customer retention. Suleymanova (2013) and Batista (2013) stated that mobile applications are the "new face of client engagement for the healthcare industry". She stressed the importance of mobile applications healthcare insurance providers as a carefully created mobile applications that can be used as a promotional initiative to comply with the suggestive number of preventative checkups, finding a doctor, looking up doctor's fees and ambulant treatment, finding benefits' related information and account claims. Quick insights can also be seen, and information can be retrieved on understanding treatment options most effective for a certain illness/disease, identifying the side effects of a drug, which all can contribute to lower the cost of healthcare. Some self-management practices are also employed to facilitate access of various resources used to achieve well-being and fitness goals of the individuals and their long-term health goals and the management of their conditions will slowly reduce costs of health insurance companies as the chances of patients getting sick due to lifestyle problems will potentially be reduced. For instance, there are devices that track bio-signals of the users and if they notice any sudden changes, they send a warning for critical health complications (Suleymanova 2013).

Mobile health applications are useful to analyze lifestyle of the customer to check if he/she is pursuing a healthy lifestyle which includes diet, exercise, good nutrition etc. which is useful for the retention of the customers. By offering users good updates of their active lifestyle through applications usage can have impact on acquiring new customers and retention of existing customers. Customer intimacy also plays an important role in this area. Execution of business more consistently is also about supplying reliable products or services while leading the market in pricing. The ability to quickly respond to customers' needs has a positive effect on customer, and a strong correlation with healthcare costs reduction. The level of trust is another important aspect that determines the commitment towards customer. The collection and analysis of large amount data related to insurance companies if accurately reported can find the underlying patterns and can provide guidance in many cases (Ra et al., 2017). This type of information provided by one central health-related mobile application may have the potential to help insurance providers retain customers. However, there is little to no research in this area knowing that it is only in recent years, the data reporting practices has just efficiently started; although not to the extend we wish to see.

\section{RESEARCH QUESTIONS}

In an effort to contribute to the research gap regarding the business value aspects of healthcare mobile applications such as customer retention, this research study seeks to examine the number of health insurance providers who are utilizing/requiring health-related mobile applications for smart devices and the relationship between their use and customer retention., There are three research questions.

1. How many health care insurance providers/payers plan or utilized/require mobile applications?

2. To what extend the healthcare payers/providers use mobile application's sources? And how does it impact the healthcare insurance payers'/providers' cost and members' retention? 
3. Will there be a s significant impact and difference between the use of mobile applications and customer retention?

Concluding for the proposed research questions above, a proposed research model has been drawn in figure 1 .

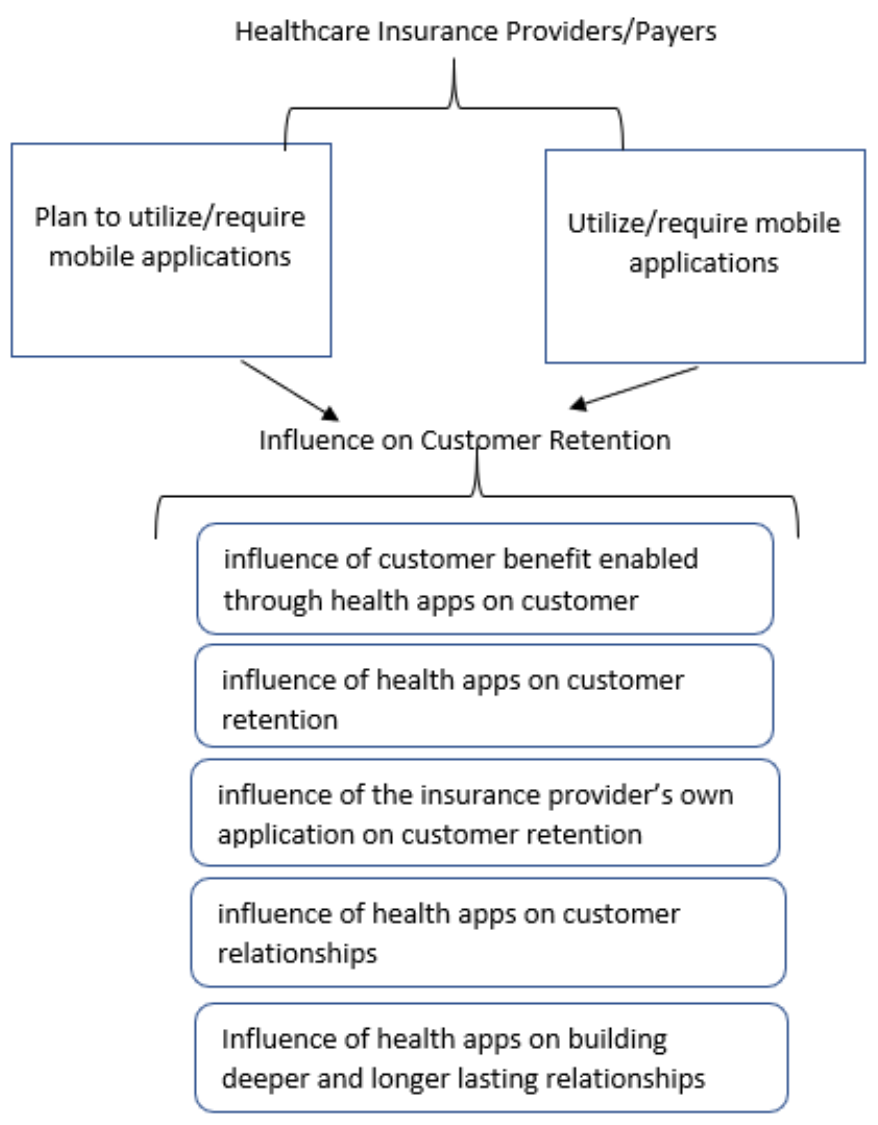

Figure 1. Proposed Research Model

\section{RESEARCH METHODOLOGY}

The goal of this research study is to investigate the number of healthcare insurance providers/payers using mobile applications for smart devices and analyzes the significance between the use of the mobile application for smart devices, their customer retention, and the impact on both. This research randomly surveyed a total of 50 participants of which only 41 respondents completed the survey. Due to corporate policies and privacy limitations, only 50 members within three healthcare payer systems could complete the questionnaire. The survey was distributed to various levels of workers, including local and regional managers, insurance agents, and claims departments, working for private insurance payers as well as the government. The randomly selected sample population for this study have in-depth understanding of the use of mobile apps to help and benefit their members' population health, in addition to knowledge on the effect and impact of healthcare applications on health insurance companies and members alike. In order to reach out the respondents of the three health insurance companies (both government sponsored and private health insurance companies) direct contact via intranet, face-to-face interactions, and email were used to distribute the 
questionnaire. Respondents contacted via email were the contact point to other employees within the three healthcare organizations. Approximately 50 employees received the request to complete the survey. The response rate was $82 \%$ as 41 health insurance employees completed the questionnaire. Data was analyzed using SPSS analytics platform to perform descriptive statistics, factor analysis to understand the correlation between the healthcare insurance payers' use or willingness to use mobile healthcare applications and customers' retention, and KMO (Kaiser-Meyer-Olkin) score to see how suited the data is for the factor analysis performed.

It is important to state that the survey was a Self-created survey of ten questions. Due to the gap in the literature, a self-created survey of 10 questions was established and administered to the participants.

\section{RESULTS}

As mentioned above, a total of 41 respondents have successfully completed the survey. Out of these 41 respondents, 32 are employed by firms offering government sponsored health insurance like Medicare, Medicaid while 9 are employed by firms offering private health insurance like Highmark and Capital Blue Cross. The results from competitiveness question indicate that most participants perceive the competition among the health insurance companies is very high while only a few believe it is just average. Less than half of the respondents (18) have indicated that the firm for which they work do not currently offer a health-related mobile application. Also, out of these 21 respondents, 16 have indicated that they work for government sponsored health insurance provider while 5 work for private health insurance provider. 20 respondents indicated that their company is offering at least one mobile health application. Out of these 20,15 work for government sponsored health insurance provider and 5 work for private health insurance provider. 31 respondents answered yes when asked about whether their firm is planning to offer a mobile health application. Also, respondents whose firms offer a mobile application currently are planning to offer another mobile health application as well. One of the observations from the result is that mobile health application that provide health related information are the most common in the market.

Another analysis was performed to identify the business value where aspects such as cost reduction and customer retention are evaluated. For this purposes, common factor analysis was conducted and KMO (Kaiser-Meyer-Olkin Test) score for this sample revealed a score of 0.675 which is a mediocre and close to acceptable value, but not sufficiently enough. The researchers were hoping for a KMO value that is between 0.8 and 1 . However, this is understandable as the researcher were not be able within the time assigned for the study to expose the questionnaire to a larger sample to collect larger data set. Hence, this could be a potential for conducting a follow-up study with a larger data set. Additionally, the customer retention has a mean of 3.42 which indicates that the relation between mobile health application and customer retention is higher than the average. The questions 'influence of health apps on customer retention' $(\mathrm{F}(1,28)=7.560, \mathrm{p}=.007)$, 'influence of the insurance provider's own application on customer retention' $(\mathrm{F}(1,31)=6.9, \mathrm{p}=.01))$, 'influence of health apps on building deeper and longer lasting relationships' $(\mathrm{F}(1,40)=5.90, \mathrm{p}=.03)$, 'influence of customer benefit enabled through health apps on customer retention' $(\mathrm{F}(1,37)=5.082, \mathrm{p}=.015)$ and 'influence of health apps on customer relationships' $(\mathrm{F}(1,43)=6.08, \mathrm{p}=.02)$ have been answered significantly more optimistic by respondents that work for private health insurance providers. Significant relationships were found between the mobile applications and customer retention.

\section{CONCLUSIONS, LIMITATIONS, AND FUTURE RESEARCH}

Health-related mobile applications for smart devices offer a lot of potentials to the patient, physicians, and healthcare insurance providers. There are plenty of ways for health payers to make use of mobile applications and increase customer value. One of the keyways to gain business value for health insurance companies is that they can integrate various concepts of mobile health applications into one application. This one stop shop can help customer to provide all types of information and data. This research focused on the relationship between of health-related mobile applications for smart devices and customer retention. The results imply that this research is important because it provides exploratory information for insurance providers, mobile application developers, and patients. Additionally, it adds the limited body of research regarding the business value or relationship between of health-related mobile applications for smart devices and customer retention. 


\section{Issues in Information Systems}

Volume 21, Issue 1, pp. 30-36, 2020

It is important to note that this research is not without limitations. First, the research is limited in size as only three healthcare insurance provides were obtained and only 41 employees completed the questionnaire. This research did not collect demographic data. Future research should address these limitations. Additionally, future research should focus on examining additional business value factors such as cost reduction. Moreover, while the business value from these health applications are promising, it might not always be the case as government regulations regarding healthcare keep changing and so this topic needs to be considered with caution. In conclusion, this research provides an important foundation for additional research on business value or relationship between of health-related mobile applications for smart devices and customer retention.

\section{REFERENCES}

Amadeo, K. (2020). The rising cost of health care by year and its causes. Retrieved from: https://www.thebalance.com/causes-of-rising-healthcare-costs-4064878

Batista, M.A. (2013). The future of smartphones in health care. AMA Journal of Ethics, 15(11):947-950.

Clemens, E. (2017). Using Smartphones and health apps to change and manage health behaviors: A populationbased survey. Retrieved from https://www.ncbi.nlm.nih.gov/

Collins, A. (2014). Health care dealmakers should consider mobile tools. Mergers \& Acquisitions Report, 29(7) 17.

Daecher, A., Cotteleer, M., \& Holdowsky, J. (2018). The internet of things: A technical primer. Deloitte. Retrieved from: https://www2.deloitte.com/insights/us/en/focus/internet-of-things/technical-primer.html.

Egbedion, B., Wimmer, H., Rebman, C., \& Powell, L. (2018). Building an application for custom mobile medication reminders in healthcare: an exploratory study. Issues in Information Systems, 19(3)139-149. Retrieved from: http://www.iacis.org/iis/2018/3_iis_2018_139-149.pdf

Ehrenhard, M., et al. (2016). Unlocking how start-ups create business value with mobile applications: Development of an App-enabled business innovation cycle. Technological Forecast and Social Change. doi: 10.1016/j.techfor.2016.011

Hamed, P. Salehi (2018). Smartphone for healthcare communication. Journal of Healthcare Communications. Retrieved from: https://healthcare-communications.imedpub.com/smartphone-for-healthcarecommunication.php?aid $=23228$

Hilfiker, S. W., Santana, S., Freedman, M., \& Harris, L. M. (2019). There's a gap between digital health information and users - let's close it. Information Services \& Use, 39(1/2), 15-22. Retrieved from: https://doi.org/10.3233/ISU-180032

Hoffmann T, Jansen J, \& Glasziou, P. (2018). The importance and challenges of shared decision making in older people with multimorbidity. PLoS Med. 15(3): e1002530. https://doi.org/10.1371/journal.pmed.1002530

King, W. (2017). The healthcare internet of things. Pharmaceutical Executive, 37(1), 34-35.

Koohang, A., Paliszkiewicz, J., Nord, J.N., Paullet, K., \& Underwood, T. (2019). predictors of success in security and data protection awareness Of Mobile Devices: Trust and Privacy. Issues in Information Systems, 20(1) $1-11$.

Liu, C., Zhu, Q., Holroyd, K.A. \& Seng, E.K. (2011). Status and trends of mobile-health applications for iOS devices: A developer's perspective. The Journal of Systems and Software, 84(2011), 2022-2033.

Latulippe, K., Hamel, C. \& Giroux, D. (2017). Social health inequalities and eHealth: a literature review with qualitative synthesis of theoretical and empirical studies, Journal of Med Internet Res, 19(4), e136 http://doi.org/10.2196/jmir.6731.

Mackert, M., Mabry-Flynn, A., Champlin, S., Donovan, E.E., \& Pounders, K. (2016). Health literacy and health information technology adoption: the potential for a new digital divide. Journal of Medical Internet Research, 18(10):e264. Retrieved from: https://www.ncbi.nlm.nih.gov/pmc/articles/PMC5069402/

MacLeod, J. (2018). Digital health technology in diabetes care: It's transforming the way people with diabetes communicate with personal health care teams and treat and manage their disease. Today's Dietitian, 20(8), $40-43$.

Marakhimov, A., \& Joo, J. (2017). Consumer adaptation and infusion of wearable devices for healthcare. Computers in Human Behavior, 76, 135-148.

Pew Research Center A. (n.d.). Internet/broadband fact sheet. Retrieved from http://www.pewinternet.org/factsheet/internet-broadband/. 


\section{Issues in Information Systems}

Volume 21, Issue 1, pp. 30-36, 2020

Pew Research Center B. (n.d.). The smartphone difference. Retrieved from: http://www.pewinternet.org/2015/04/01/us-smartphone-use-in-2015/.

Pinchot, J. (2018). A mobile app development project model emphasizing communication skills, teamwork, and user-centered design. Issues in Information Systems, 19(3) 22-32.

Polansky, J., Leslie, \& G. Heimann (2018) The great American search for healthcare information. Retrieved from: https://www. webershandwick.com/wp-content/uploads/2018/11/Healthcare-Info-Search-Report.pdf.

Preetinder, S., Ashwini, K., \& Tejkaran, S. (2012). Distraction: An assessment of smartphone usage in health care work settings. US National Library of Medicine, 2012(5), 105-114.

Rama, A., \& Saritha, P. (2019). Customer retention scenario in private life insurance services. Journal of the Insurance Institute of India, 6(3), 44-52.

Ray, R., Gilbert, K.L,.\& Sewell, A. A. (2016). Mobile technology as a conduit for reducing obesity-related health disparities. Issues in Race and Society, 4(1), 98-119.

Sampat, B., \& Prabhakar, B. (2017). Privacy risks and security threats in mHealth apps. Journal of International Technology \& Information Management, 26(4), 126-153.

Sidow, L. (2018). Global app store records shattered yet again in Q1 2018. App Annie. Retrieved from: https://www.appannie.com/en/insights/market-data/q1-2018-apps-record-downloads-spend/

Steven, L., \& Ryan, G. (2016). Use of smartphones and mobile devices in hospitalized patients: Untapped opportunities for inpatient engagement. Retrieved from https://www.ncbi.nlm.nih.gov/

Suleymanova, A. (2013). Empowering patients: How mobile apps are influencing the future of health plan customer loyalty. Health Management Technology, 34(2), 10-11.

Talwar, Y. K., Karthikeyan, S., Bindra, N., \& Medhi, B. (2016). Smartphone' - a user-friendly device to deliver affordable healthcare - a practical paradigm. Journal of Health and Medical Informatics, 7(3), 1-7.

Tovino, S. A. (2019). Going rogue: Mobile research applications and the right to privacy. Notre Dame Law Review. 95(1), 155-209. 